BURA/2438/3432

July 17, 2009

IC/HEP/09-3

IPPP/09/47

DCPT/09/94

FERMILAB-FN-0836-APC

MPP-2009-82

VT-IPNAS-09-07

\title{
Study of Low-Energy Neutrino Factory at the Fermilab to DUSEL Baseline
}

This note constitutes a Letter of Interest to study the physics capabilities of, and to develop an implementation plan for, a neutrino physics program based on a Low-Energy Neutrino Factory at Fermilab providing a veam to a detector at the Deep Underground Science and Engineering Laboratory.

It has been over ten years since the discovery of neutrino oscillations [1] established the existence of neutrino masses and leptonic mixing. Neutrino oscillations thus provide the first evidence of particle physics beyond the Standard Model. Most of the present neutrino oscillation data are well described by the $3 v$ mixing model. While a number of the parameters in this model have already been measured, there are several key parameters that are still unknown, namely, the absolute neutrino mass scale, the precise value of the mixing angles, the $\mathrm{CP}$ phase $\delta$ and hence the presence or absence of observable CP-violation in the neutrino sector. Future measurements of these parameters are crucial to advance our understanding of the origin of neutrino masses and of the nature of flavor in the lepton sector. The ultimate goal of a program to study neutrino oscillations goes beyond a first measurement of parameters, and includes a systematic search for clues about the underlying physics responsible for the tiny neutrino masses, and, hopefully, the origin of the observed flavor structure in the Standard Model, as well as the possible source of the observed matter-antimatter asymmetry in the Universe. To achieve this goal will almost certainly require precision measurements that go well beyond the presently foreseen program.

One of the most promising experimental approaches to achieve some of the goals mentioned above is to build a Neutrino Factory and its corresponding detector. The Neutrino Factory produces neutrino beams from muons which have been accelerated to an energy of, for example, $25 \mathrm{GeV}$. The muons are stored in a race-track shaped decay ring and then decay along the straight sections of the ring. Since the decay of the muon is well understood, the systematic uncertainties associated with a neutrino beam produced in this manner are very small. Beam diagnostics in the decay ring and a specially designed near detector further reduce the systematic uncertainties of the neutrino beam produced at the Neutrino Factory. In addition since the muon (anti-muon) decays produce both muon and anti-electron neutrinos (anti-muon and electron neutrinos), many oscillation channels are accessible from a Neutrino Factory, further extending the reach in the oscillation parameter space. Over the last decade there have been a number of studies [2-5] that have explored the discovery reach of Neutrino Factories in the small mixing angle, $\theta_{13}$, and its capability to determine the mass hierarchy and determine if $\mathrm{CP}$ is violated in leptons through observation of phase parameter, $\delta$. The most recent study to be completed [6], the International scoping study of a future Neutrino Factory and super-beam facility (the ISS), studied the physics capabilities of various future neutrino facilities: super-beam, $\beta$-Beam and Neutrino Factory and has determined that the Neutrino Factory with an energy of $\sim 25 \mathrm{GeV}$ has the best discovery reach for small values of $\sin ^{2} 2 \theta_{13}$, reaching an ultimate sensitivity of between $10^{-5}$ and $10^{-4}$. However, for larger values of $\sin ^{2} 2 \theta_{13}\left(>10^{-3}\right)$, the sensitivity of other experimental approaches is competitive to that of the $25 \mathrm{GeV}$ Neutrino Factory. 
The wide-band neutrino beam (WBB) produced at Fermilab and directed towards DUSEL [7] is one such competitor. For the case where $\sin ^{2} 2 \theta_{13}$ is large, initial studies have shown that a Low-Energy Neutrino Factory [8-10] with an energy of, for example, $4 \mathrm{GeV}$, may be both cost-effective and offers exquisite sensitivity. The required baseline for a Low-Energy Neutrino Factory matches Fermilab to DUSEL and, therefore, its physics potential and implementation should be studied in the context of DUSEL along with those for the WBB.

The Low-Energy Neutrino Factory, which stores muons with energies between 4 and $5 \mathrm{GeV}$, requires a detector technology that can detect low energy muons and at the same time determine their charge. Recently ideas for such a detector based on a fully active calorimeter within a potentially affordable large volume magnet have emerged. These ideas strongly motivate consideration of the Low-Energy Neutrino Factory. Initial studies suggest that a Neutrino Factory with energy of about $4 \mathrm{GeV}$ operating at a single baseline of approximately $1300 \mathrm{~km}$ would enable very precise measurements of the neutrino mixing parameters to be made while simultaneously having a discovery reach in $\sin ^{2} 2 \theta_{13}$ of approximately $10^{-4}$. The ongoing Neutrino Factory Study, the International Design Study for a Neutrino Factory (IDS-NF) [11] is developing the baseline high-energy $(25 \mathrm{GeV})$ Neutrino Factory and is considering as an option in the case of large $\theta_{13}$, the Low-Energy Neutrino Factory. Since up to the final acceleration and decay rings the $25 \mathrm{GeV}$ Neutrino Factory and the low energy option are identical, the IDS will provide the accelerator physics and engineering foundation for both the $25 \mathrm{GeV}$ Neutrino Factory and the low-energy option.

The totally active scintillator detector (TASD) has been proposed for the Low-Energy Neutrino Factory. The results from a first study of its expected detector performance were described in the ISS Detector Working Group Report [12]. Using a TASD for neutrino physics is not new; examples are KamLAND, which has been operating for several years, and NOvA. Note that unlike KamLAND or NOvA, the TASD is required to be magnetized and to have a segmentation that is approximately 10 times that of NOvA. Magnetization of such a large volume $\left(>30,000 \mathrm{~m}^{3}\right)$ is the main technical challenge in designing a TASD for a Neutrino Factory, although R\&D to reduce the detector cost (driven in part by the large channel count) is also needed.

The Neutrino Factory TASD we are considering consists of long plastic scintillator bars with a triangular cross-section arranged in planes which make measurements of the $\mathrm{x}$ and $\mathrm{y}$ coordinates of hits along tracks. Optimization of the cell cross section needs further study since a true triangular cross section results in tracking anomalies at the corners of the triangle. The scintillator bars have a length of $15 \mathrm{~m}$ and the triangular cross-section has a base of $3 \mathrm{~cm}$ and a height of $1.5 \mathrm{~cm}$. Our design is an extrapolation of the MINERvA experiment [11], which in turn was an extrapolation of the D0 preshower detectors [12]. We are considering a detector mass of approximately $35 \mathrm{kTon}$ (dimensions 15 X 15 X 150 $\mathrm{m})$ which results in a $20 \mathrm{kT}$ on fiducial mass. We believe that an air-core solenoid can produce the field $(0.5$ Tesla) required to determine the muon charge with the necessary charge mis-ID rate.

As was mentioned above, magnetizing the large detector volume presents the main technical challenge for the proposed detector. Conventional room temperature magnets are ruled out due to their prohibitively expensive power consumption, and conventional superconducting magnets are believed to be too expensive due to the cost of the enormous cryostats that would be needed. In order to eliminate the cryostat, we have investigated a concept based on the superconducting transmission line (STL) that was developed for the Very Large Hadron Collider superferric magnets [14]. The solenoid windings now consist of this superconducting cable which is confined in its own cryostat. Each solenoid (10 are required for the full detector) consists of 150 turns and requires $7500 \mathrm{~m}$ of cable. Since there is no large vacuum vessel, and thus no large vacuum loads, the cost of the solenoids based on the STL is expected to be significantly smaller than the conventional solenoid alternative. 
We believe that there is a great deal of synergy between the WBB study for Fermilab to DUSEL and a Low-Energy Neutrino Factory. Underground engineering studies at Fermilab are appropriate for both a WBB and the muon decay ring for the Low-Energy Neutrino Factory. Cavern design and engineering studies at the DUSEL site are similar for both the Low-Energy Neutrino Factory and the initial phase of the WBB physics program ( $25 \mathrm{kTon}$ ) which require caverns of similar size. In addition if the R\&D for large LAr detectors is successful and this detector technology becomes an option for the WBB, LAr would also be an option for the detector for the Low-Energy Neutrino Factory since the magnetization concepts being considered for TASD are likely also applicable for a large LAr detector.

The Low-Energy Neutrino Factory serving a long-baseline neutrino physics program utilizing the Fermilab to DUSEL baseline offers a number of very exciting opportunities: not only does the Low Energy Neutrino Factory have the potential to discover leptonic CP violation and to make the definitive measurements of the neutrino mixing parameters that are required to guide the development of the underlying physics, but the intense neutrino beam would have the potential to serve a charged-lepton flavor violation program of exquisite sensitivity and to be a step on the way to a multi-TeV muon-antimuon colliding beam facility. Such a program would establish the US at the forefront of neutrino and lepton-flavor physics and provide a route for FNAL to develop the energy-frontier accelerator required to follow the LHC. We urge that the study of the Low Energy Neutrino Factory serving a neutrino detector in the Deep Underground Science and Engineering Laboratory be energetically pursued.

Paul Kyberd

Malcolm Ellis

Alan Bross

Steve Geer

Olga Mena

Ken Long

Silvia Pascoli

Enrique Fernandez Martinez

Kirk McDonald

Patrick Huber

\author{
Brunel University \\ Brunel University \\ Fermilab \\ Fermilab \\ IFIC, University of Valencia \\ Imperial College \\ IPPP, Durham University \\ Max-Planck Institute for Physics \\ Princeton University \\ Virginia Tech
}

\section{References}

[1] Y.Ashi e et al., “ A Measurement of Atmospheric Neutrino Oscillation Parameters by Super-Kamiokande I,” Phys. Rev. D 71 (2005) 112005.

[2] S. Geer, H. Schellman (Eds.) hep-ex/0008064.

[3] N. Holtkamp and D. Finley (Eds.) Fermilab-Pub-00/108-E.

[4] Autin B, Blondel A, Ellis J. CERN-99-02 ; DeRujula A, Gavela M, Hernandez P. Nucl. Phys. B547 (1999) 21.

[5] Y. Mori J. Phys. G: Nucl. Part. Phys. 29 (2003) 1527:1536.

[6] RAL-TR-2007-019, 023, 024

[7] V. Barger et al., (FNAL-BNL Long Baseline Neutrino Experiment Study), FERMILAB-0801-AD-E, BNL-77973-2007-IR, FERMILAB-APC, May 2007. 109pp. Preprint: arXiv:0705.4396 [hep-ph]

[8] S. Geer, O. Mena, S. Pascoli, Phys. Rev. D75, 093001 (2007)

[9] A. Bross, M. Ellis, S. Geer, O. Mena, S. Pascoli, Phys. Rev. D77, 093012 (2008)

[10] C. Ankenbrandt et al. FERMILAB-PUB-09-001-APC (2009).

[11] https://www.ids-nf.org/wiki/FrontPage

[12] JINST 4:T05001,2009; arXiv:0712.4129v1

[13] K. S. McFarland, Eur. Phys. J. A 24S2, 187 (2005).

[14] P. Baringer et al. (D0 Collaboration), Nucl. Instrum. Methods Phys. Res., Sect. A 469, 295 (2001).

[15] Ambrosio et al., Report No. Fermilab-TM-2149, 2001. 